\title{
Long noncoding RNA CASC2 predicts the prognosis of glioma patients and functions as a suppressor for gliomas by suppressing $\mathrm{Wnt} / \beta$-catenin signaling pathway
}

\author{
This article was published in the following Dove Press journal: \\ Neuropsychiatric Disease and Treatment \\ II July 2017 \\ Number of times this article has been viewed
}

\author{
Ronglin Wang ${ }^{1, *}$ \\ Yuqian $\mathrm{Li}^{1, *}$ \\ Gang Zhu',* \\ Bo Tian' \\ Wen Zeng' \\ Yang Yang ${ }^{2}$ \\ Zhihong $\mathrm{Li}^{\prime}$ \\ 'Department of Neurosurgery, \\ Tangdu Hospital, The Fourth Military \\ Medical University, ${ }^{2}$ Department of \\ Neurosurgery, The 45 I th hospital of \\ PLA, Xi'an, Shaanxi, People's Republic \\ of China \\ *These authors contributed equally \\ to this work
}

Correspondence: Zhihong Li

Department of Neurosurgery, Tangdu Hospital, The Fourth Military Medical University, Xi'an, Shaanxi 710038,

People's Republic of China

Tel +86 I3709।8 3909

Fax +86029 847। 782I

Email 729793045@qq.com

Yang Yang

Department of Neurosurgery, The 45 I th hospital of PLA, Xi'an, Shaanxi 710054,

People's Republic of China

Tel +86 I77 925I 0809

Email yyfmmu@I26.com

\begin{abstract}
Background: Previous studies have demonstrated that long noncoding RNA cancer susceptibility candidate 2 (lncRNA CASC2) is frequently downregulated in several types of tumors and functions as a tumor-suppressive factor. However, the clinical significance and function of CASC2 in human glioma remain largely unknown. The purpose of this study was to identify the clinical values of CASC2, as well as investigate the potential molecular mechanisms in glioma.
\end{abstract}

Methods: This retrospective study first analyzed the expression levels of CASC2 using quantitative real-time polymerase chain reaction. Then, CASC2 expression levels were associated with various clinicopathologic characteristics and the survival rate of patients with glioma. Finally, the function and underlying molecular mechanisms of CASC2 in human glioma were investigated in U251 cell line.

Results: By quantitative real-time polymerase chain reaction analysis, our data showed that CASC2 expression was significantly downregulated in glioma tissues and cell lines (U87 and U251) compared to adjacent normal brain tissues or normal human astrocytes. Moreover, its expression negatively correlated with tumor grade in glioma patients. Furthermore, KaplanMeier curves with log-rank analysis revealed a close correlation between downregulated CASC2 and shorter survival time in glioma patients. In addition, Cox regression analysis indicated that CASC2 could be considered as an independent risk factor for poor prognosis. Finally, in vitro experiment demonstrated that CASC2 overexpression remarkably suppressed glioma cell proliferation, migration, and invasion through suppressing Wnt/ $\beta$-catenin signaling pathway.

Conclusion: This study suggested that CASC2 may potentially serve as a valuable diagnostic and prognostic biomarker and a therapeutic target for glioma patients.

Keywords: glioma, lncRNA, CASC2, biomarker, Wnt/ $\beta$-catenin

\section{Introduction}

Human glioma, arising from astrocytes or astroglial precursors, is the most frequent and malignant primary brain tumor in the central nervous system. The overall incidence of glioma is about 4-5/100,000 persons per year. ${ }^{1}$ Although the improvements in tumor diagnosis and treatment including neurosurgery, radiotherapy, and chemotherapy are tremendous, the prognosis of glioma patients still remains poor. ${ }^{2}$ The 5 -year survival rate in low-grade (grade I-II) glioma patients is 30\%-70\%, while the median survival time of glioblastoma multiform (World Health Organization grade IV) is from 9 to 12 months. Therefore, it is necessary to elucidate the specific molecular mechanisms that mediate 
the initiation and progression of glioma and identify novel precise diagnostic, prognostic, and therapeutic targets to improve the efficacy of clinical antiglioma management.

Human genome sequence data implied that a much larger proportion of the genome is transcribed to produce long noncoding RNAs, exceeding 200 nucleotides in length, with little protein coding ability. They are involved in the regulation of gene expression at different levels, affecting multiple cellular processes including cell differentiation, stem cell maintenance, and epithelial-mesenchymal transition. ${ }^{3-5}$ Accumulating evidence has strongly suggested that many IncRNAs function as oncogenes and/or tumor suppressors in tumor initiation and development. ${ }^{6}$ These findings make those lncRNAs potential diagnostic and therapeutic targets in tumor management. Cancer susceptibility candidate 2 (CASC2) is a novel lncRNA first identified as a downregulated gene and it plays a role as a tumor suppressor gene in endometrial cancer. ${ }^{7}$ Recently, several reports indicate that CASC2 plays a tumor-suppressive role in various human malignancies, including non-small cell lung cancer, gastric carcinoma, colorectal carcinoma, renal cell carcinoma, and glioma. ${ }^{8-11}$ However, little is known about the clinical significance as well as the potential molecular mechanisms of CASC2 in glioma, which are important questions remaining to be further elucidated.

To investigate these issues, the expression level of CASC2 was measured in human glioma tissues and cell lines compared with adjacent normal brain tissues (NBT) and normal human astrocytes by quantitative real-time polymerase chain reaction (qRT-PCR) assay. Further, we clarified the association of CASC2 with clinicopathologic factors or prognosis of glioma patients. Moreover, we investigated the effect of CASC2 on glioma cell proliferation, migration, and invasion and explored the potential molecular mechanisms. Our findings suggest that CASC2 may function as a novel prognostic predictor and a valuable therapeutic target for glioma.

\section{Materials and methods Human tissue samples}

Human glioma tissues and adjacent NBT were obtained from 47 glioma patients who were treated with surgical resection between July 2010 and October 2011 in the Department of Neurosurgery at Tangdu Hospital of Fourth Military Medical University (Xi'an, Shaanxi, People's Republic of China). No enrolled patients in our study received chemotherapy or radiotherapy prior to surgery. All procedures performed in the study were approved by the Research Ethics Committee of Tangdu Hospital, and written informed consent was obtained from all patients. All collected tissue samples were immediately frozen in liquid nitrogen until total RNAs or proteins were extracted.

\section{Cell culture}

Normal human astrocytes (NHA) and human glioma cell lines U251 and U87 were purchased from Beijing Zhongyuan Company (Beijing, People's Republic of China). All cells were maintained in Dulbecco's Modified Eagle's Medium with high glucose and sodium pyruvate supplemented with $10 \%$ heat-inactivated fetal bovine serum (Thermo Fisher Scientific, Waltham, MA, USA) and antibiotics (100 units/mL penicillin and $100 \mathrm{mg} / \mathrm{mL}$ streptomycin) at $37^{\circ} \mathrm{C}$ in a humidified incubator with $5 \% \mathrm{CO}_{2}$.

\section{Lentivirus infection}

Lentivirus-carrying hsa-lnc RNA CASC2 or negative control (NC) was purchased from Obio Technology (Shanghai, People's Republic of China). The U251 cells were transduced with lentivirus $48 \mathrm{~h}$ before using.

\section{Chemicals and antibodies}

The antibodies used for Western blotting were anti- $\beta$-catenin (1:1,000, ab134175; Abcam, Cambridge, UK), anti-C-Myc (1:1,000, 13987; Cell Signaling, Beverly, MA, USA), antiCyclin-D1 (1:1,000, 2978; Cell Signaling), and anti- $\beta$-actin (1:2,000, 4967; Cell Signaling). The chemical used was SKL2001 (selleck, s8320, Houston, TX, USA).

\section{RNA preparation, reverse transcription, and $\mathrm{QRT}$-PCR}

Total RNA was extracted from the frozen glioma tissues and non-neoplastic brain using TRIzol ${ }^{\circledR}$ reagent (Thermo Fisher Scientific) referring to the manufacturer's recommendation. Then cDNA synthesis and qRT-PCR were performed using an IQTM 5 Real-Time PCR Detection System (Thermo Fisher Scientific) to examine the expression levels in glioma tissues and cell lines with U6 as a normalizing control. Quantitative analysis of the change in expression levels of CASC2 was tested and normalized using the $2-\Delta \Delta \mathrm{Ct}$ method relative to U6. Expression levels of $\beta$-catenin, Cyclin D1, c-Myc, and IDH1 were normalized using the $2-\Delta \Delta C$ t method relative to GAPDH. The primers used are listed in Table 1.

\section{Pyrosequencing}

IDH1 codon 132 was amplified by PCR using the specific primers shown in Table 1. Pyrosequencing was conducted on a Qiagen PyroMark Q24 system (Qiagen GmbH, Hilden, 
Table I Sequence of primers in RT-qPCR

\begin{tabular}{ll}
\hline Names & Sequences 5' -3' $^{\prime}$ \\
\hline CASC2-F & GCACATTGGACGGTGTTTCC \\
CASC2-R & CCCAGTCCTTCACAGGTCAC \\
U6-F & CTCGCTTCGGCAGCACA \\
U6-R & AACGCTTCACGAATTTGCT \\
$\beta$-catenin & AAAATGGCAGTGCGTTTAG \\
$\beta$-catenin & TTTGAAGGCAGTCTGTCGTA \\
c-Myc-F & TGCCCATTTGGGGACACTTC \\
c-Myc-R & TACGGCTGCACCGAGTCGT \\
CyclinDI-F & CGCCCCACCCCTCCAG \\
CyclinDI-R & CCGCCCAGACCCTCAGACT \\
GAPDH-F & CGGAGTCAACGGATTTGGTCGTAT \\
GAPDH-R & AGCCTTCTCCATGGTGGTGAAGAC \\
IDHI-F & GCTTGTGAGTGGATGGGTAAAA \\
IDHI-R & ATTGCCAACATGACTTACTTGATC \\
\hline
\end{tabular}

Note: "F" and "R" represent forward and reverse primers, respectively.

Abbreviations: CASC2, cancer susceptibility candidate 2; GAPDH, glyceraldehyde 3-phosphate dehydrogenase; IDHI, isocitrate dehydrogenase I; RT-qPCR, reverse transcription quantitative polymerase chain reaction.

Germany) according to the manufacturer's protocol. Ten microliters of PCR product, $28 \mu \mathrm{L}$ of water, $2 \mu \mathrm{L}$ of Streptavidin Sepharose High-Performance beads, and $40 \mu \mathrm{L}$ of PyroMark binding buffer were mixed and agitated for $10 \mathrm{~min}$ at 1,400 rpm to bind PCR products to the beads. ${ }^{12}$ The beads were washed in $70 \%$ ethanol, denatured with denaturation buffer, and washed in $50 \mathrm{~mL}$ washing buffer. Purified DNA samples released from the beads were annealed to the sequencing primer 5'-TGGGTAAAACCTATCATC-3'. Pyrograms were manually interpreted and evaluated using the PyroMark Q24 software.

\section{Cell proliferation}

The U251 cells were seeded at about 3,000 cells per well in 96-well plates in the logarithmic phase of growth. Cell Counting Kit-8 assay (CCK8; Dojindo Laboratories, Kumamoto, Japan) was used to evaluate cell proliferation. After adding $10 \mu \mathrm{L}$ of CCK8 solution, the OD value was measured at $450 \mathrm{~nm}$ every $24 \mathrm{~h}$, referring to the instructions of the manufacturer.

\section{Wound healing assay}

The U251 cells were grown in six-well plates until they reached $90 \%$ confluence. A scratch was then applied to form wounded gaps using a $20 \mu \mathrm{L}$ pellet tip. Then, we washed the cells twice with PBS and observed cell migration to the wounded region using Nikon microscopy at the time point we designated.

\section{Transwell assay of cell invasion}

To evaluate the invasion, $1 \times 10^{5} \mathrm{U} 251$ cells were suspended in $200 \mu \mathrm{L}$ serum-free medium and seeded into the upper chamber of transwell inserts precoated with Matrigel (Corning Incorporated, Corning, NY, USA). Eight hundred microliters of complete medium was added to the lower chambers of each well. After incubation for $24 \mathrm{~h}$, the migrated cells were fixed with methanol and stained with $0.1 \%$ crystal violet. Then the stained cells in five random fields were counted using a microscope.

\section{Western blot analysis}

The cells were washed with cold PBS three times and suspended in lysis buffer ( $100 \mu \mathrm{L}$ lysis buffer per $6 \mathrm{~cm}$ plate). The lysed samples were separated by $10 \%$ sodium dodecyl sulfate-polyacrylamide gel electrophoresis and transferred to polyvinylidene fluoride (PVDF) membranes. The PVDF membranes were blocked at room temperature with 5\% non-fat milk for $2 \mathrm{~h}$ and incubated with the corresponding antibodies with gentle shaking at $4^{\circ} \mathrm{C}$ for $12 \mathrm{~h}$. Then, the PVDF membranes were incubated with a secondary antibody conjugating horseradish peroxidase with gentle shaking for $2 \mathrm{~h}$ at room temperature. The protein bands were visualized using electrochemiluminescence.

\section{Statistical analysis}

All collected data in our study were analyzed using the software SPSS version 20.0 (IBM Corporation, Armonk, NY, USA) and are expressed as mean \pm SD. The difference between two independent groups was compared by independent sample $t$-test. One-way analysis of variance was performed to compare the difference among the three groups. Pearson's chi-square test was used to assess the relationship between CASC2 expression and clinicopathologic parameters. The overall survival rate of the patients was calculated with Kaplan-Meier method, and data were analyzed by the log-rank test. A multivariate Cox regression was carried out to calculate the hazard ratio (HR) and the 95\% confidence interval (CI) and evaluate the association between CASC2 expression and survival. Differences were considered statistically significant when $P<0.05$.

\section{Compliance with ethical standards}

All procedures performed in this study were approved by the Research Ethics Committee of Tangdu Hospital of Fourth Military Medical University, and informed consent was obtained from each of the patients.

\section{Results}

CASC2 expression was significantly downregulated in human glioma tissues and cell lines. We first examined 

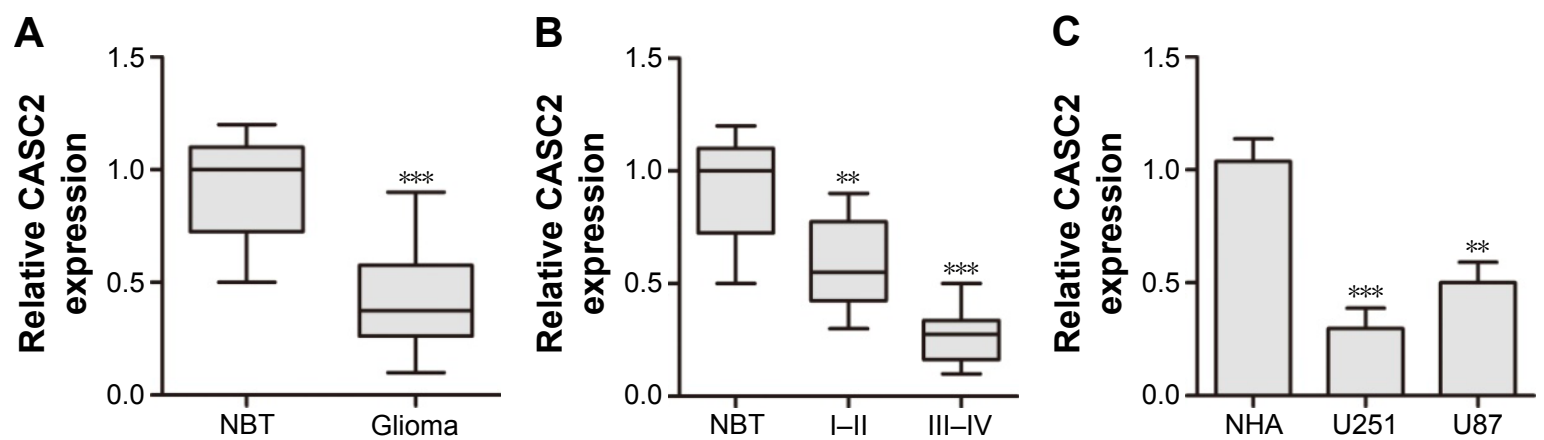

Figure I CASC2 expression was significantly downregulated in human glioma tissues and cell lines.

Notes: (A) CASC2 expression was downregulated in glioma tissues compared to adjacent NBT. (B) CASC2 expression was much lower in grade III-IV than that in grade I-II. (C) CASC2 expression was downregulated in two glioma cell lines (U87 and U25I) compared to NHA. $* * P<0.0$ I and $* * * P<0.00 \mathrm{I}$ compared with control.

Abbreviations: CASC2, cancer susceptibility candidate 2; NBT, normal brain tissues; NHA, normal human astrocytes.

CASC2 expression in glioma tissues and NBT by qRT-PCR. Our data showed that the expression level of CASC2 was greatly downregulated in glioma tissues compared to NBT ( $0.43 \pm 0.23$ vs $0.91 \pm 0.22, P<0.01$; Figure $1 \mathrm{~A})$. In addition, CASC2 expression in high-grade (grade III-IV) glioma was significantly lower than that in low-grade (grade I-II) glioma $(0.27 \pm 0.13$ vs $0.59 \pm 0.20, P<0.05$; Figure $1 \mathrm{~B})$, indicating that CASC2 expression level was inversely related to advanced histologic grade of glioma. Then, the expression level of CASC2 in two glioma cell lines (U87, U251) and NHA was evaluated by qRT-PCR. Consistent with the tendency in tissues, our findings indicated that CASC2 expression in U87 and U251 cell lines was significantly decreased compared to that in NHA $(P<0.01$; Figure 1C).

\section{Correlations between CASC2 expression and clinicopathologic characteristics of glioma}

To further investigate the role of CASC2 in determining the clinical significance of glioma, we evaluated its expression with different clinicopathologic features in 47 glioma patients. Twenty-five cases with CASC2 expression less than the median expression level (mean expression value 0.39 ) were assigned to the low expression group (mean expression value 0.24 ). By contrast, the remaining 22 cases with CASC2 expression above the median expression level were assigned to the high expression group (mean expression value 0.55 ). Our results revealed that low expression level of CASC2 significantly correlated with advanced tumor grade $(P=0.024)$ and low Karnofsky performance score (KPS; $P=0.010$ ), but there were no significant associations with age, gender, tumor location, and wild-type IDH1 ${ }^{\mathrm{R} 132 \mathrm{H}}(P=0.319,0.763,0.341$, and 0.557 , respectively; Table 2).

\section{Low expression level of CASC2 was significantly associated with poorer prognosis in glioma patients}

The prognostic significance of CASC2 expression was investigated in patients with glioma. Kaplan-Meier curves with log-rank analysis were used to explore whether CASC2 expression level was associated with the overall survival rates of glioma patients. Our results revealed that patients with low expression level of CASC2 had distinctly shorter survival time than patients with high CASC2 expression

Table 2 Correlation between IncRNA CASC2 expression level and clinicopathological characteristics in glioma patients

\begin{tabular}{|c|c|c|c|c|}
\hline \multirow[t]{2}{*}{ Features } & \multirow{2}{*}{$\begin{array}{l}\text { No of } \\
\text { patients }\end{array}$} & \multicolumn{2}{|c|}{ CASC2 expression } & \multirow[t]{2}{*}{$P$-value } \\
\hline & & High (22) & Low (25) & \\
\hline Age (year) & & & & 0.319 \\
\hline$<45$ & 25 & 10 & 15 & \\
\hline$\geq 45$ & 22 & 12 & 10 & \\
\hline Gender & & & & 0.763 \\
\hline Male & 31 & 15 & 16 & \\
\hline Female & 16 & 7 & 9 & \\
\hline Tumor grade & & & & 0.024 \\
\hline I-II & 26 & 16 & 10 & \\
\hline III-IV & 21 & 6 & 15 & \\
\hline KPS & & & & 0.010 \\
\hline$\geq 80$ & 27 & 17 & 10 & \\
\hline$<80$ & 20 & 5 & 15 & \\
\hline Tumor location & & & & $0.34 I$ \\
\hline Frontal & 19 & 11 & 8 & \\
\hline Parietal & 9 & 5 & 4 & \\
\hline Temporal & 14 & 5 & 9 & \\
\hline Others & 5 & I & 4 & \\
\hline $\mathrm{IDH}^{\mathrm{R} \mid 32 \mathrm{H}}$ & & & & 0.557 \\
\hline Mutant & 11 & 6 & 5 & \\
\hline Wild-type & 36 & 16 & 20 & \\
\hline
\end{tabular}

Abbreviations: $\mathrm{CASC} 2$, cancer susceptibility candidate 2; IDHI, isocitrate dehydrogenases I; KPS, Karnofsky performance score. 


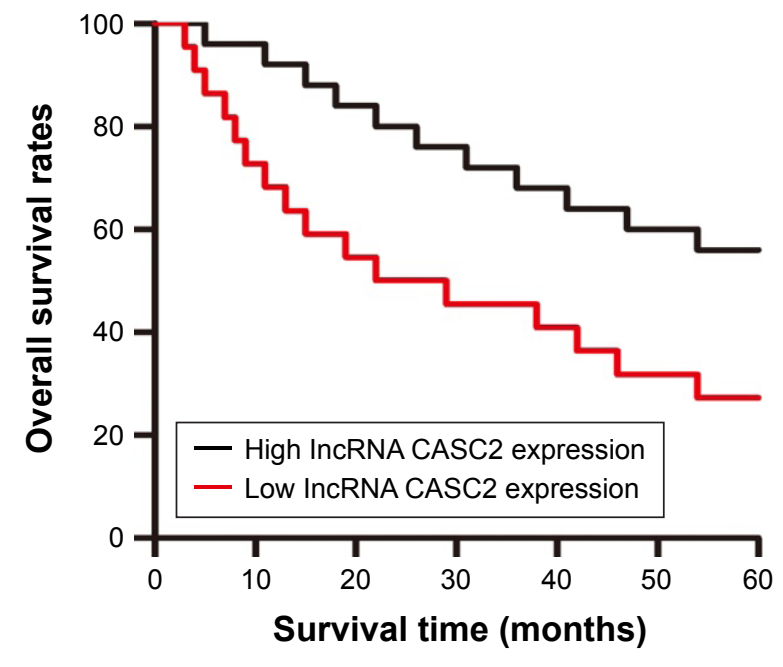

Figure 2 CASC2 expression was associated with poor prognosis in glioma patients. Notes: Kaplan-Meier curves of the overall survival of glioma patients with high and low expression levels of CASC2. The overall survival rate in patients with low CASC2 expression (red line) was significantly lower than that in patients with high CASC2 expression (black line).

Abbreviation: CASC2, cancer susceptibility candidate 2 .

level, showing a close correlation between low expression of CASC2 and poor prognosis in these glioma patients $(P<0.05$; Figure 2). Then, univariate analysis using a Cox proportional hazard regression model was performed to evaluate whether CASC2 expression level and other clinicopathologic features were independent prognostic markers for glioma patients. Our data indicated that CASC 2 expression level $(\mathrm{HR}=2.032$, 95\% CI: $1.186-3.592, P=0.035)$, tumor grade ( $\mathrm{HR}=1.864$, 95\% CI: $1.012-3.531, P=0.034), \mathrm{KPS}(\mathrm{HR}=1.853,95 \% \mathrm{CI}$ : $1.067-3.038, P=0.032)$, and $\mathrm{IDH} 1^{\mathrm{R} 132 \mathrm{H}}(\mathrm{HR}=1.652,95 \%$ CI: $0.932-2.796, P=0.044)$ were independent predictors of prognosis in glioma patients (Table 3). After adjusting for clinicopathologic characteristics, CASC2 expression level ( $\mathrm{HR}=3.162,95 \% \mathrm{CI}: 1.865-5.892, P=0.023)$, tumor grade $(\mathrm{HR}=2.682,95 \% \mathrm{CI}: 1.413-5.054, P=0.014)$, KPS (HR $=2.256,95 \%$ CI: $1.462-4.057, P=0.021)$, and $\mathrm{IDH}^{\mathrm{R} 132 \mathrm{H}}$ (HR $=2.134,95 \%$ CI: $\left.1.238-3.986, P=0.019\right)$ remained significantly correlated with prognosis of glioma patients (Table 3).

\section{CASC2 functions as a tumor suppressor by suppressing $\mathrm{Wnt} / \beta$-catenin signaling activity}

We first detected the activity of Wnt/ $\beta$-catenin signaling pathway in our samples. The data indicated that the mRNA levels of $\beta$-catenin, Cyclin D1, and c-Myc were greatly increased in glioma tissues compared to adjacent NBT (Figure $3 \mathrm{~A}-\mathrm{C}$ ). These findings revealed $\mathrm{Wnt} / \beta$-catenin signaling was activated in glioma tissues which had low CASC2 expression. Therefore, we hypothesized that the activity of Wnt/ $\beta$-catenin signaling pathway may be regulated by CASC2. Then, U251 cells were treated with NC lentiviral vectors, CASC2 lentiviral vectors, and CASC2 + SKL2001, respectively. SKL2001 is an activator of $\mathrm{Wnt} / \beta$-catenin signaling pathway. ${ }^{13} \mathrm{We}$ found that the overexpression of CASC2 could remarkably reduce nuclear $\beta$-catenin and its downstream targets, while the effect could be reversed by SKL2001 (Figure 3D). CCK8 assay, wound healing assay, and transwell assay were carried out to detect the impact of those interventions on glioma cell proliferation, migration, and invasion. Our results showed that CASC 2 overexpression

Table 3 Univariate and multivariate analyses of different clinicopathologic variables and IncRNA CASC2 expression in glioma patients

\begin{tabular}{|c|c|c|c|c|}
\hline \multirow[t]{2}{*}{ Characteristics } & \multicolumn{2}{|l|}{ Univariate analysis } & \multicolumn{2}{|l|}{ Multivariate analysis } \\
\hline & HR (95\% CI) & $P$-value & HR (95\% CI) & P-value \\
\hline Age ( $<45$ vs $\geq 45$ years) & I.I55 (0.532-2.362) & 0.534 & I.357 (0.632-2.749) & 0.344 \\
\hline Gender (female vs male) & $1.054(0.498-2.372)$ & 0.583 & $0.674(0.378-1.472)$ & 0.473 \\
\hline CASC2 expression (low vs high) & $2.032(1.186-3.592)$ & 0.035 & $3.162(1.865-5.892)$ & 0.023 \\
\hline Tumor grade (I-II vs III-IV) & $1.864(1.012-3.531)$ & 0.034 & $2.682(1.4 \mid 3-415.054)$ & 0.014 \\
\hline KPS $(<80$ vs $\geq 80)$ & $1.853(1.067-3.038)$ & 0.032 & $2.256(1.462-464.057)$ & 0.021 \\
\hline IDHII ${ }^{R / 32 H}$ (mutant vs wild-type) & $1.652(0.932-2.796)$ & 0.044 & $2.134(1.238-3.986)$ & 0.019 \\
\hline \multicolumn{5}{|l|}{ Tumor location } \\
\hline Parietal vs frontal & $\mathrm{I} .503(0.536-3.24 \mathrm{I})$ & 0.435 & $1.589(0.52 \mathrm{I}-0.336)$ & 0.336 \\
\hline Temporal vs frontal & $1.221(0.592-2.634)$ & 0.593 & $1.429(0.432-2.973)$ & 0.345 \\
\hline Occipital vs frontal & $0.583(0.224-1.596)$ & 0.539 & $0.842(0.302-I .842)$ & 0.678 \\
\hline Others vs frontal & $1.045(0.334-2.268)$ & 0.656 & I.337 (0.442-2.888) & 0.432 \\
\hline Tumor size $(\leq 4$ vs $>4 \mathrm{~cm})$ & I. $135(0.468-2.435)$ & 0.685 & I.I $55(0.286-2.324)$ & 0.688 \\
\hline
\end{tabular}

Abbreviations: CASC2, cancer susceptibility candidate 2; HR, hazard ratio; KPS, Karnofsky performance score; IDHI, isocitrate dehydrogenase I. 
A

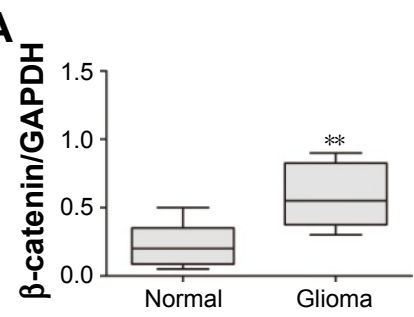

B

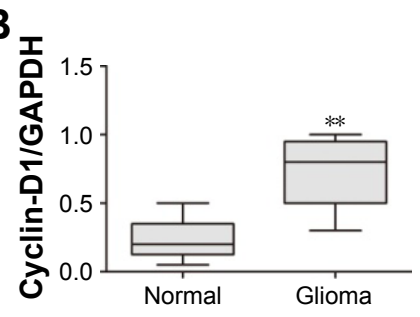

C

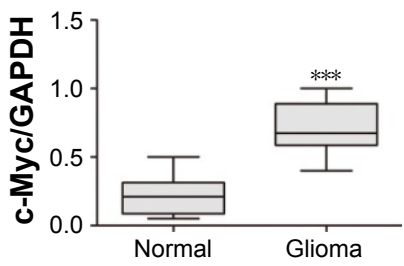

D

E
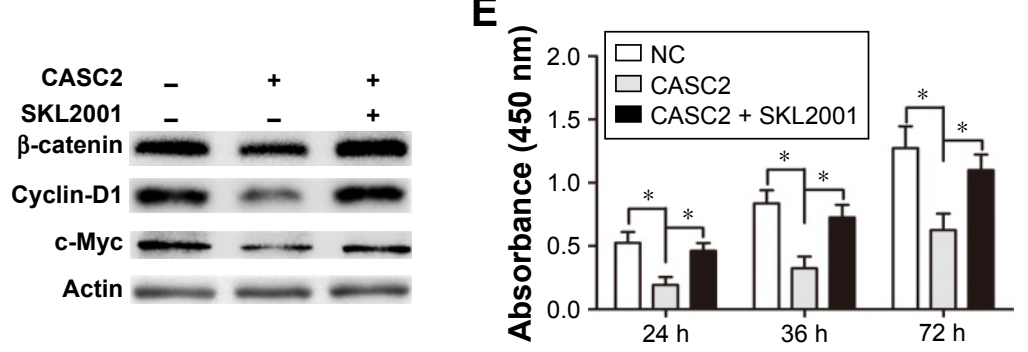

\section{F}

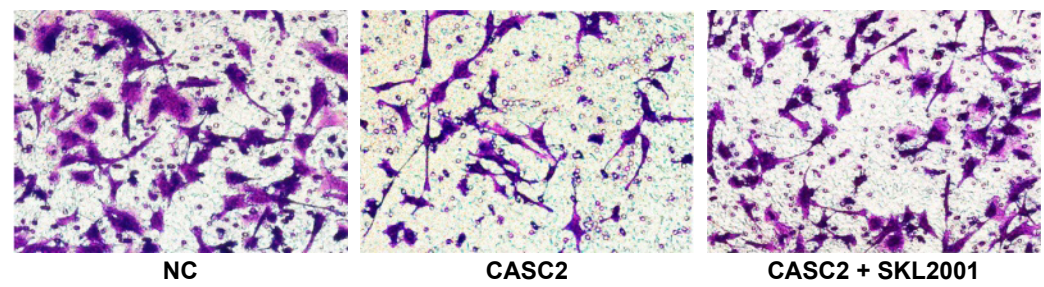

G
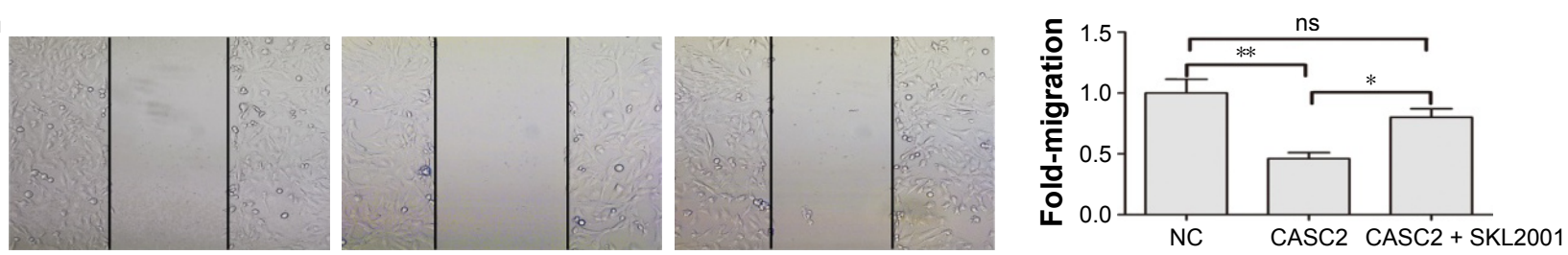

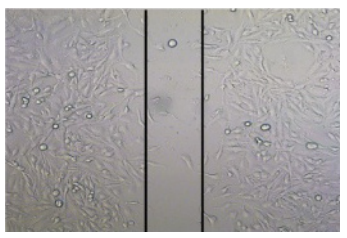

NC

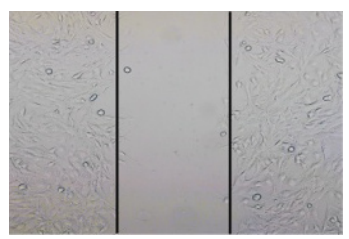

CASC2

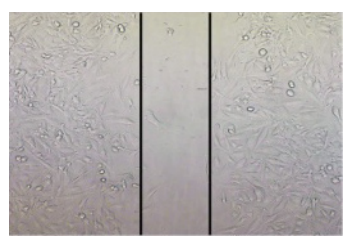

CASC2 + SKL2001

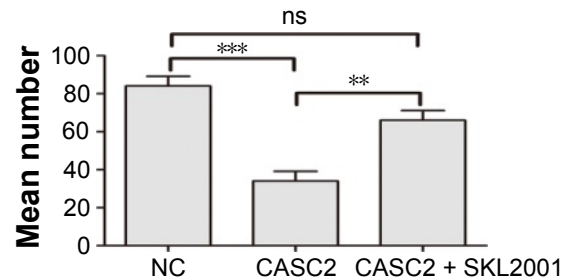

Figure 3 CASC2 functions as a tumor suppressor by suppressing $W n t / \beta$-catenin signaling activity.

Notes: (A-C) $\beta$-catenin, Cyclin-DI, and c-Myc were upregulated in glioma tissues compared with adjacent normal brain tissues, as found by qRT-PCR analysis. (D) Overexpression of CASC2 suppressed the protein expression of $\beta$-catenin, Cyclin-DI, and c-Myc in U25I cells and the effect could be reversed by SKL200 I. (E-G) CASC2 overexpression restrained the proliferation, migration, and invasion capacity in $\mathrm{U} 25 \mathrm{I}$ cells and $\mathrm{SKL} 200 \mathrm{I}$ could reverse the effect of $\mathrm{CASC2}$. $* \mathrm{P}<0.05$, $* * P<0.0 \mathrm{I}$, $* * * P<0.00 \mathrm{I}$. Abbreviations: CASC2, cancer susceptibility candidate 2; NC, negative control; ns, no significance; qRT-PCR, quantitative real-time polymerase chain reaction.

dramatically suppressed cell proliferation, migration, and invasion, whereas the tumor-suppressive effect of CASC2 on U251 cells was reversed by SKL2001, indicating that Wnt/ $\beta$-catenin signaling is the downstream mechanism of CASC2 (Figure 3E-G). Taken together, we confirmed that CASC2 partly inhibited malignant progression of glioma by suppressing $\mathrm{Wnt} / \beta$-catenin signaling activity. To further study the function of CASC2, we knocked down the expression of CASC2 in U251 cells (Figure S1A). The expression of $\beta$-catenin, cyclin D1 and c-Myc are slightly increased in the knock down group (Figure S1B). Proliferation and invasion of U251 cell were tested and it seems that there was no significant difference between the knock down group and the $\mathrm{NC}$ group (Figure S1C, D). The basic level of CASC2 was very low in U251 cells, the knock down of CASC2 may have little influence on the proliferation and migration of glioma cells.

\section{Discussion}

Due to the lack of specific targets, it is rather difficult to cure glioma with current therapies, especially glioblastoma 
multiform. Thus, the identification of novel reliable biomarkers as diagnostic, prognostic, and therapeutic targets is of great significance for malignant glioma patients. Accumulating evidence indicates that lncRNAs might be involved in a variety of biologic and pathologic activities including cell proliferation, differentiation, migration, cell cycle regulation, and so on. ${ }^{14,15}$ Furthermore, a close association between lncRNAs expression and prognosis of patients with cancer has also been revealed. Ellinger et al discovered that lncRNA ZNF180-2 is a prognostic biomarker in patients with renal cell carcinoma. ${ }^{16}$ Cheng et al found that lncRNA AB073614 promotes tumorigenesis and predicts poor prognosis in ovarian cancer. ${ }^{17}$ In addition, Wang et al demonstrated that lncRNA HOXA11-AS played a crucial role in glioma development and acted as an independent prognostic factor in glioma patients. ${ }^{18}$ However, so far, only a handful of lncRNAs have been reported to function as independent prognostic factors for glioma.

LncRNA CASC2 is a novel lncRNA transcript which plays a tumor-suppressive role in various human malignancies. He et al found that CASC2 indicates a poor prognosis and regulates cell proliferation in non-small cell lung cancer. ${ }^{9}$ However, little is known about the clinical significance of CASC2 in glioma. Here, we aimed to investigate the clinical role and prognostic value of CASC2 in glioma development, as well as the underlying molecular mechanisms.

In this study, we first detected CASC2 expression in human glioma tissues and adjacent NBT, as well as in glioma cell lines and NHA. The result of qRT-PCR analysis showed a significant decrease of CASC 2 expression in glioma tissues and cell lines, and it is highly related to histologic tumor grade. Furthermore, multivariate Cox proportional hazards regression model analysis identified low CASC2 expression as an independent indicator of poor prognosis. These results imply that CASC2 may act as a diagnostic and prognostic biomarker as well as a tumor suppressor in glioma.

Recently, CASC2 was reported to be involved in some classical signaling pathways to function as a tumor suppressor. ${ }^{11,19} \mathrm{Wnt} / \beta$-catenin signaling pathway is a highly conserved molecular mechanism which was reported to play a crucial role in a variety of human malignancies, including ovarian cancer, hepatocellular carcinoma, breast cancer, and colorectal cancer, by activating its downstream targets including Cyclin D1, c-Myc, GSK-3, and so on. ${ }^{20-23}$ To explore the association between CASC 2 and $\mathrm{Wnt} / \beta$-catenin signaling pathway, we measured the mRNA level of $\beta$-catenin, Cyclin D1, and c-Myc in glioma tissues and found that their levels increased significantly when compared to adjacent NBT.
The discovery indicated a reciprocal relation between CASC2 and Wnt/ $\beta$-catenin signaling pathway. Furthermore, U251 cells were transduced with lentivirus encoding CASC 2 and we found the protein level of $\beta$-catenin, Cyclin D1, and c-Myc remarkably reduced, indicating $\mathrm{Wnt} / \beta$-catenin signaling pathway was inversely regulated by CASC 2 in glioma cells. Also, SKL2001, a Wnt/ $\beta$-catenin activator, can reverse the effect of CASC2. Then, we explored whether CASC2 played the tumor-suppressive role by suppressing $\mathrm{Wnt} / \beta$-catenin signaling pathway in glioma. Our results showed that overexpression of CASC2 can hinder U251 cells proliferation, migration, and invasion, while the tumor-suppressive effect was reversed after treatment with SKL2001. Taken together, these findings strongly suggest that the tumor-suppressive effect of CASC2 is attributed to its ability to suppress the activation of $\mathrm{Wnt} / \beta$-catenin signaling pathway in glioma.

In conclusion, our study provided potent evidence to confirm that CASC2 has potential value for diagnosing and predicting the clinical outcomes of glioma patients. Additionally, it can serve as a tumor suppressor in glioma by downregulating Wnt/ $\beta$-catenin signaling pathway, suggesting CASC 2 might be a potential therapeutic target in the treatment of glioma. These findings may facilitate understanding of tumorigenesis of glioma and provide further evidence for CASC2 to be a potential diagnostic, prognostic, and therapeutic candidate in glioma.

\section{Acknowledgment}

This study was supported by the National Natural Science Funds for Distinguished Young Scholar (Grant No: 81501047).

\section{Disclosure}

The authors report no conflicts of interest in this work.

\section{References}

1. Siegel R, Ma J, Zou Z, Jemal A. Cancer statistics. 2014. CA Cancer J Clin. 2014;64(1):9-29.

2. Boele FW, Rooney AG, Grant R, Klein M. Psychiatric symptoms in glioma patients: from diagnosis to management. Neuropsychiatr Dis Treat. 2015;11:1413-1420.

3. Li D, Ji Y, Wang F, et al. Regulation of crucial lncRNAs in differentiation of chicken embryonic stem cells to spermatogonia stem cells. Anim Genet. 2017;48(2):191-204.

4. Wang P, Xue Y, Han Y, et al. The STAT3-binding long noncoding RNA lnc-DC controls human dendritic cell differentiation. Science. 2014; 344(6181):310-313.

5. Zhou S, Ding F, Gu X. Non-coding RNAs as Emerging Regulators of Neural Injury Responses and Regeneration. Neurosci Bull. 2016;32(3): 253-264.

6. Wang Z, Dai J, Hu N, et al. Identification of new susceptibility loci for gastric non-cardia adenocarcinoma: pooled results from two Chinese genome-wide association studies. Gut. 2017;66(4):581-587. 
7. Baldinu P, Cossu A, Manca A, et al. CASC2a gene is down-regulated in endometrial cancer. Anticancer Res. 2007;27(1A):235-243.

8. Cao Y, Xu R, Xu X, Zhou Y, Cui L, He X. Downregulation of IncRNA CASC2 by microRNA-21 increases the proliferation and migration of renal cell carcinoma cells. Mol Med Rep. 2016;14(1):1019-1025.

9. He X, Liu Z, Su J, et al. Low expression of long noncoding RNA CASC2 indicates a poor prognosis and regulates cell proliferation in non-small cell lung cancer. Tumour Biol. 2016;37(7):9503-9510.

10. Huang G, Wu X, Li S, Xu X, Zhu H, Chen X. The long noncoding RNA CASC2 functions as a competing endogenous RNA by sponging miR-18a in colorectal cancer. Sci Rep. 2016;6:26524.

11. Li P, Xue WJ, Feng Y, Mao QS. Long non-coding RNA CASC2 suppresses the proliferation of gastric cancer cells by regulating the MAPK signaling pathway. Am J Transl Res. 2016;8(8):3522-3529.

12. Cykowski MD, Allen RA, Fung KM, Harmon MA, Dunn ST. Pyrosequencing of IDH1 and IDH2 mutations in brain tumors and nonneoplastic conditions. Diagn Mol Pathol. 2012;21(4):214-220.

13. Gwak J, Hwang SG, Park HS, et al. Small molecule-based disruption of the Axin/beta-catenin protein complex regulates mesenchymal stem cell differentiation. Cell Res. 2012;22(1):237-247.

14. Bina P, Kakhki MP, Sahraian MA, Behmanesh M. The expression of lnc-IL-7R long non-coding RNA dramatically correlated with soluble and membrane-bound isoforms of IL-7Ra gene in multiple sclerosis patients. Neurosci Lett. 2017;642:174-178.

15. Zhang W, Zhou Y, Ding Y. Lnc-DC mediates the over-maturation of decidual dendritic cells and induces the increase in Th1 cells in preeclampsia. Am J Reprod Immunol. 2017.

16. Ellinger J, Alam J, Rothenburg J, et al. The long non-coding RNA lncZNF180-2 is a prognostic biomarker in patients with clear cell renal cell carcinoma. Am J Cancer Res. 2015;5(9):2799-2807.
17. Cheng Z, Guo J, Chen L, Luo N, Yang W, Qu X. A long noncoding RNA AB073614 promotes tumorigenesis and predicts poor prognosis in ovarian cancer. Oncotarget. 2015;6(28):25381-25389.

18. Wang Q, Zhang J, Liu Y, et al. A novel cell cycle-associated lncRNA, HOXA11-AS, is transcribed from the 5-prime end of the HOXA transcript and is a biomarker of progression in glioma. Cancer Lett. 2016; 373(2):251-259.

19. Liao Y, Shen L, Zhao H, et al. LncRNA CASC2 interacts with miR181a to modulate glioma growth and resistance to TMZ Through PTEN Pathway. J Cell Biochem. 2017;118(7):1889-1899.

20. Chen MW, Yang ST, Chien MH, et al. The STAT3-miRNA-92-Wnt signaling pathway regulates spheroid formation and malignant progression in ovarian cancer. Cancer Res. 2017;77(8):1955-1967.

21. Gkretsi V, Stylianou A, Stylianopoulos T. Vasodilator-Stimulated Phosphoprotein (VASP) depletion from breast cancer MDA-MB-231 cells inhibits tumor spheroid invasion through downregulation of Migfilin, beta-catenin and urokinase-plasminogen activator (uPA). Exp Cell Res. 2017;352(2):281-292.

22. Li H, Zhang J, Lee MJ, Yu GR, Han X, Kim DG. OIP5, a target of miR-15b-5p, regulates hepatocellular carcinoma growth and metastasis through the AKT/mTORC1 and beta-catenin signaling pathways. Oncotarget. 2017;8(11):18129-18144.

23. Liu K, Fan J, Wu J. Forkhead Box Protein J1 (FOXJ1) is overexpressed in colorectal cancer and promotes nuclear translocation of beta-Catenin in SW620 Cells. Med Sci Monit. 2017;23:856-866. 


\section{Supplementary material}
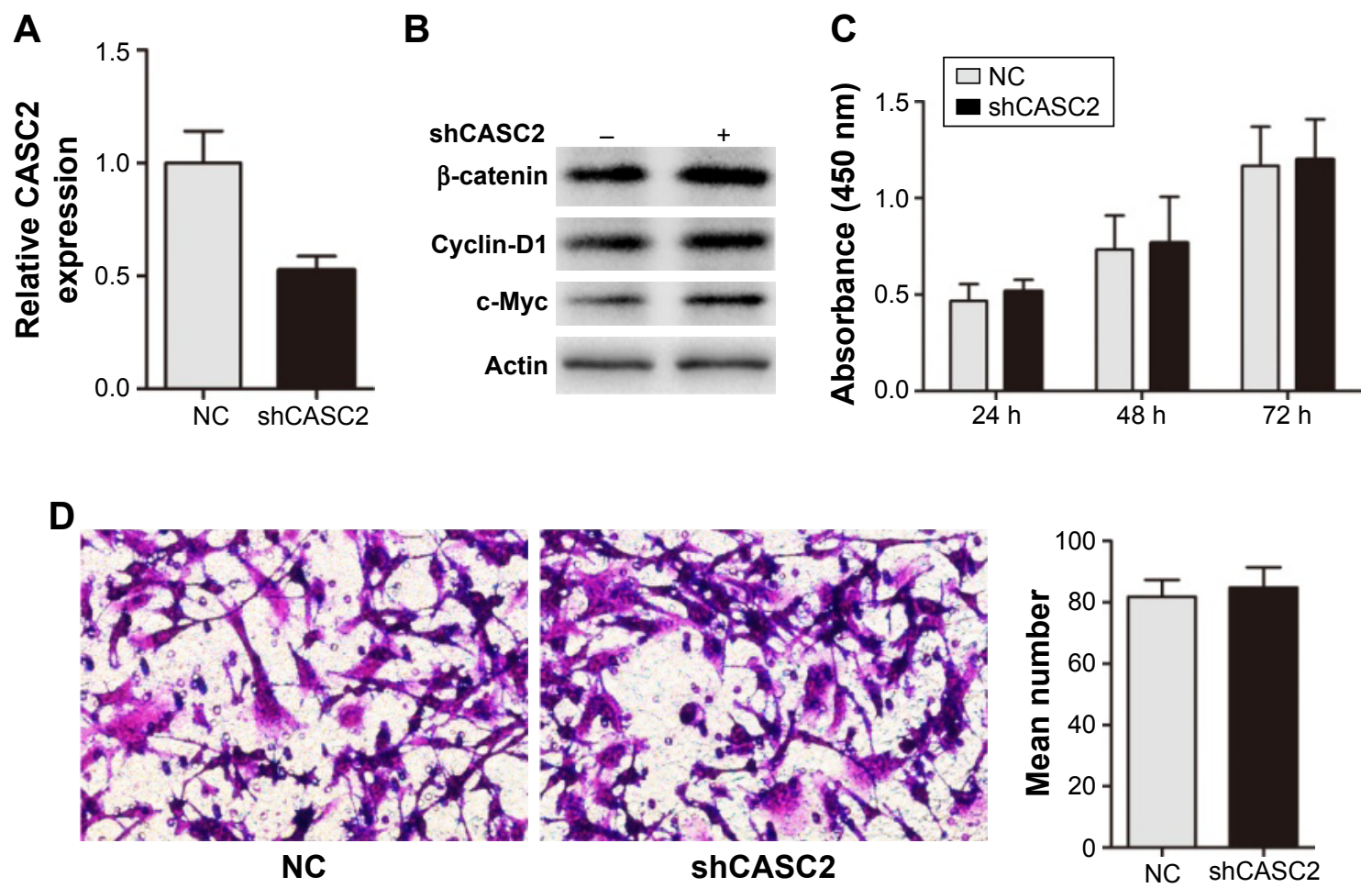

Figure SI The downregulation of CASC2 has little influence on proliferation and migration of U25I cells.

Notes: (A) CASC2 expression was downregulated when transfected with shCASC2 in U25I cell line. (B) $\beta$-catenin, Cyclin-DI, and c-Myc were upregulated when transfected with shCASC2. (C) The shCASC2 group had no significant difference in proliferation when compared with the NC group. (D) The shCASC2 group had no significant difference in invasion when compared with the NC group.

Abbreviations: CASC2, cancer susceptibility candidate 2; NC, negative control.

\section{Publish your work in this journal}

Neuropsychiatric Disease and Treatment is an international, peerreviewed journal of clinical therapeutics and pharmacology focusing on concise rapid reporting of clinical or pre-clinical studies on a range of neuropsychiatric and neurological disorders. This journal is indexed on PubMed Central, the 'PsycINFO' database and CAS, and is the official journal of The International Neuropsychiatric Association (INA). The manuscript management system is completely online and includes a very quick and fair peer-review system, which is all easy to use. Visit http://www.dovepress.com/testimonials.php to read real quotes from published authors. 